Int. J. Odontostomat., 10(2):287-295, 2016.

\title{
La Invisibilidad de Estudios en Salud Oral en Mujeres en Situación de Prostitución: Una Revisión Sistemática Exploratoria
}

\author{
The Invisibility of Oral Health Studies about Women \\ in Situation of Prostitution: A Scoping Review
}

\author{
Alejandro Vásquez Hernández;; Annie M. Vivares-Builes ${ }^{* * *}$ \& Andrés A. Agudelo-Suárez
}

VÁSQUEZ, H. A.; VIVARES-BUILES, A. M. \&. AGUDELO-SUÁREZ, A. A. La invisibilidad de estudios en salud oral en mujeres en situación de prostitución: Una revisión sistemática exploratoria. Int. J. Odontostomat., 10(2):287-295, 2016.

RESUMEN: El objetivo fue describir los estudios existentes a nivel mundial relacionados con salud oral en mujeres en situación de prostitución. Para ello se realizó una revisión exploratoria (Scoping Review) sobre el tema en cuestión en estudios publicados en forma de artículos científicos originales en revistas de revisión por pares, sin restricción en el periodo de búsqueda e indexados en 4 bases de datos internacionales (PubMed, EMBASE, LILACS y SCOPUS). Descripción de las principales características de los estudios. Como resultados principales, luego de hacer el proceso de selección se encontraron 10 estudios con diferentes enfoques metodológicos (cuantitativos, cualitativos o mixtos y estudios de intervención). Seis de los estudios fueron realizados en países asiáticos (Japón y Singapur). Las temáticas abordadas fueron: manifestaciones orales del VIH, uso del condón, prácticas y actitudes frente al sexo oral. Solamente se encontró un estudio específico en salud oral realizado en Senegal el cual analiza la situación periodontal en trabajadoras sexuales con y sin VIH. Los estudios en salud oral para la población en situación de prostitución son escasos. Siendo un grupo sensible y vulnerable socialmente, se requiere fomentar la investigación en estas mujeres para identificar las posibles situaciones de desigualdad social que impactan en las condiciones de salud oral en este colectivo.

PALABRAS CLAVE: salud oral, perfil de salud, prostitución, investigación en odontología.

\section{INTRODUCCIÓN}

Las enfermedades de la cavidad oral ocupan un lugar importante dentro de la salud pública por su magnitud y severidad y porque existen desigualdades entre grupos poblacionales, que son atribuidas por un lado a los estilos de vida y por otro, a los determinantes sociales (Newton \& Bower 2005; Agudelo Suárez \& Martínez Herrera, 2009). El análisis de la situación de salud oral por lo tanto implica reconocer y visibilizar a los grupos especialmente vulnerables por su pertenencia a un grupo étnico determinado, por razones de género o por factores que afectan el acceso a servicios de salud oral tanto preventivos como curativos (Agudelo Suárez \& Martínez Herrera; Petersen \& Kwan, 2011).

Uno de los colectivos especialmente sensibles a expresar una carga de enfermedad por su exposi- ción constante a factores de riesgo por la labor que desempeñan son las mujeres en situación de prostitución o trabajadoras sexuales de calle (Amaya et al., 2005). Éstas se encuentran en una situación de especial vulnerabilidad ya que no cuentan con derechos laborales ni seguridad social y se asocian con otros problemas sociales como la droga, alcohol, violencia, el crimen y responsables del aumento de enfermedades de transmisión sexual (Belza et al., 2004; Ribando, 2010).

El trabajo sexual de calle es un fenómeno que surge como un hecho social multifactorial y como una actividad productiva que pone en evidencia las necesidades de subsistencia de algunas personas, así como aquellas relacionadas con el acceso a bienes y

\footnotetext{
* Facultad de Odontología, Fundación Universitaria Autónoma de las Américas, Medellín, Colombia.

"Facultad de Odontología, Universidad de Antioquia, Medellín, Colombia.
} 
servicios sociales (Belza et al., 2004; Ministerio de la Protección Social, 2008). De esta manera algunos de los factores que intervienen y que se han asociado con la determinación de desempeño como trabajadora sexual, incluyen la precariedad económica, la falta de oportunidades y capacidades para generar empleo y condiciones laborales precarias, falta de acceso a la educación, la exclusión social, la violencia intrafamiliar, abuso sexual, maltrato infantil y consumo de sustancias psicoactivas; Incluyendo además el desplazamiento forzado a causa del conflicto militar interno como otro factor relevante en especial en algunos países latinoaméricanos (Ministerio de la Protección Social; Ribando).

En Colombia el oficio de trabajo sexual no es un delito (Corte Constitucional, 2010), amparado en los derechos que tiene esta población, que incluye el derecho a la salud, el cual abarca una serie de libertades de controlar su propia salud y cuerpo con inclusión de la libertad sexual; posibilitando la oportunidad de decidir sobre tener o no relaciones sexuales, como, cuando, donde y con quien, elegir el compañero y la finalidad de este ejercicio ya sea recreación, comunicación, procreación, placer, satisfacción o como actividad económica (Corte Constitucional; Rua Serna, 2010). Sin embargo esta actividad laboral no está adecuadamente regulada y no se garantiza la protección y el acceso a la seguridad social y en salud a una población que se encuentra en una situación de especial vulnerabilidad, sobre todo aquellas en condición de calle (Ministerio de la Protección Social).

Estudios cualitativos y cuantitativos describen los comportamientos de alto riesgo que sufre esta población y como son determinados por factores sociales, culturales y económicos, sumado a la presencia de barreras de acceso y utilización de los servicios de promoción de la salud y prevención de la enfermedad (Carter et al., 1997; Amaya et al.; Juliano, 2005; Ministerio de la Protección Social). Sin embargo a la fecha son escasos los estudios relacionados con salud oral, que además evalúen sus determinantes y patologías asociadas, teniendo en cuenta que muchas veces la boca constituye un instrumento de trabajo actuando como una fuente de entrada y transmisión de enfermedades que contribuyen a problemas de salud pública.

De acuerdo con lo anterior el objetivo de esta revisión es describir los estudios epidemiológicos existentes a nivel mundial relacionados con salud oral en mujeres en situación de prostitución.

\section{MATERIAL Y MÉTODO}

Se realizó un estudio de revisión de la literatura científica mediante la metodología de Scoping Review (Arksey \& O'Malley, 2005; Manchado Garabito et al., 2009) la cual consiste en un proceso de mapeo de la literatura o la evidencia científica sobre un tema en cuestión y puede usarse como el paso previo a la revisión sistemática propiamente dicha (Armstrong et al., 2011). Para ello fueron consultadas cuatro bases de datos internacionales: LILACS, MEDLINE a través de PubMed, EMBASE y Scopus. La búsqueda se limitó a los artículos originales pero no se tuvo criterio de tiempo, es decir se abarcó el mayor número de evidencia disponible. Se incluyeron estudios enfocados a mujeres en situación de prostitución de calle, excluyendo población LGTBI y hombres que ejercen la prostitución. De igual forma, no se incluyeron revisiones teóricas, artículos de opinión, comentarios, editoriales, cartas o resúmenes de conferencias, descripciones o series de casos.

Como palabras clave y ecuaciones de búsqueda se tuvieron en cuenta en Español: [trabajadoras sexuales OR prostitutas OR prostitución] AND [salud Bucal/Oral OR caries dental OR cáncer bucal/oral OR enfermedad periodontal OR enfermedades bucales/ orales] y en Ingles: [oral health OR dental caries OR oral diseases OR oral cancer OR periodontal diseases] AND [sex workers OR prostitute OR prostitution].

Tras la búsqueda inicial y luego de hacer la depuración de la base de datos de aquellas referencias duplicadas se identificó 1673 referencias, luego de hacer un cribado con la lectura de los títulos y resúmenes se eliminaron 1663, por no ser relevantes con el objetivo de la revisión. En la Figura 1 se describe detalladamente el proceso de selección de los artículos incluidos en la Scoping Review.

Para el análisis de la información se incluyó un protocolo de recolección de datos, con información del autor y año de publicación, objetivo, tipo de estudio, fuente de información, tamaño de la muestra, país del estudio, principales resultados encontratos y temática en salud oral abordada en el estudio.

Teniendo en cuenta los requerimientos éticos para investigación documental, se protegen los derechos de autor, citando apropiadamente el documento de acuerdo con los Requisitos uniformes para presentación en revistas biomédicas (International Committee of Medical Journal Editors (ICMJE), 2015). 


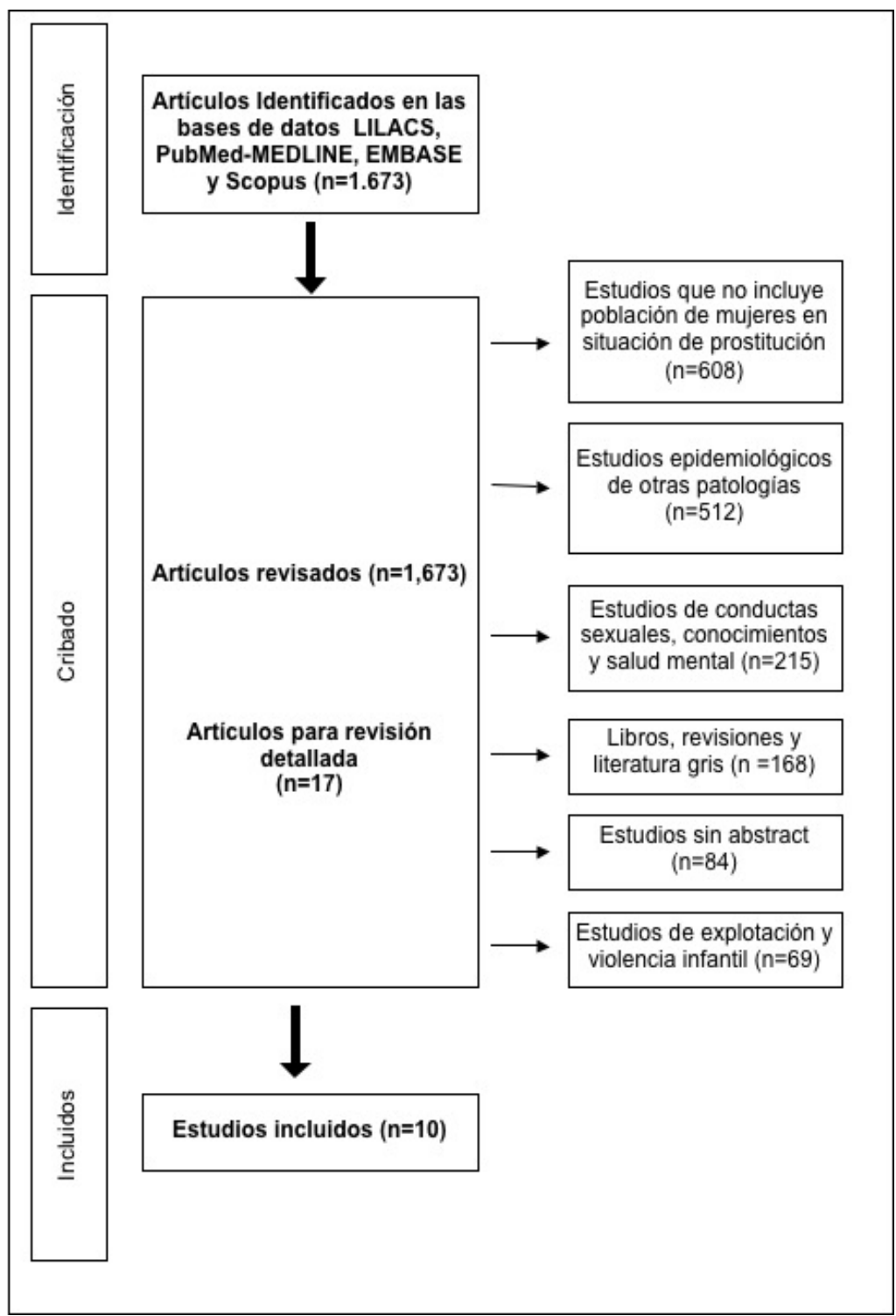

Fig. 1. Proceso de selección de artículos incluidos en la Scoping Review

\section{RESULTADOS}

En la Tabla I se describen con detalle las características de los 10 estudios incluidos en esta revisión (Ndiaye et al., 1997; Lian et al., 2000; Wong et al., 2000, 2002, 2004, 2012; de Sanjosé et al., 2002; Allen et al., 2003, 2006; Matsushita et al., 2011). La mayoría de estas investigaciones se realizaron con fuente de información primaria. En cuanto a aspectos metodológicos, 6 fueron estudios cuantitativos de carácter transversal (Ndiaye et al.; Wong et al., 2000, 2012; de Sanjosé et al.; Allen et al.; Matsushita et al., 2011), 1 estudio con enfoque cualitativo (Lian et al.), 1 estudio mixto (Allen et al., 2003), 2 estudios de intervención o evaluativos (Wong et al., 2002, 2004). En cuanto a la localizacion de los estudios, 2 estudios fueron realizados en América (México y Guyana) (Allen et al., 2003, 2006), 1 estudio en España (de Sanjosé et al.), 1 estudio en Africa (Senegal) (Ndiaye et al.) y los 6 restantes fueron conducidos en Asia (Japon y Singapur) (Lian et al., 2000; Wong et al., 2000, 2002, 2004; Matsushita et al.; Wong et al.).

Importante destacar que el componente de salud, se refería a estudios epidemiológicos, en especial el $\mathrm{VIH}$ y el papel que juegan en la transmisión de esta patología (Ndiaye et al.; Wong et al., 2000, 2012; Allen et al., 2006; Matsushita et al.). De igual forma se evaluaban aspectos concernientes a Infecciones de transmisión sexual (ITS) (Wong et al., 2012). Las temáticas referenciadas al componente de salud oral estuvieron enfocadas a la situación de salud oral relacionada con infecciones de trasmisión sexual transmitidas, diagnosticadas o con manifestaciones en tejidos orales y faríngeos (Wong et al., 2002, 2004). Se destacan los estudios relacionados a patologías específicas como aquellas desencadenadas por el Virus del Papiloma Humano (Matsushita et al.) y el Sarcoma de Kaposi. (de Sanjosé et al.).

Adicionalmente algunos estudios relacionaron las condiciones de salud con las conductas sexuales como el uso del condón durante el sexo oral (Lian et al.; Wong et al., 2000, 2002, 2004, 2012; Allen et al., 2003) y uso de fluidos orales para la detección del VIH (Allen et al., 2006). Se encontró un estudio comparativo en Senegal con referencia a la enfermedad periodontal en mujeres ensituación de prostitución infectadas y no infectadas con VIH (Ndiaye et al.), se destaca en este estudio la falta de acceso a servicios de salud general y oral, no consumo de medicamentos para el $\mathrm{VIH}$, y mayores indicadores de enfermedad gingival y periodontal en mujeres infectadas. 


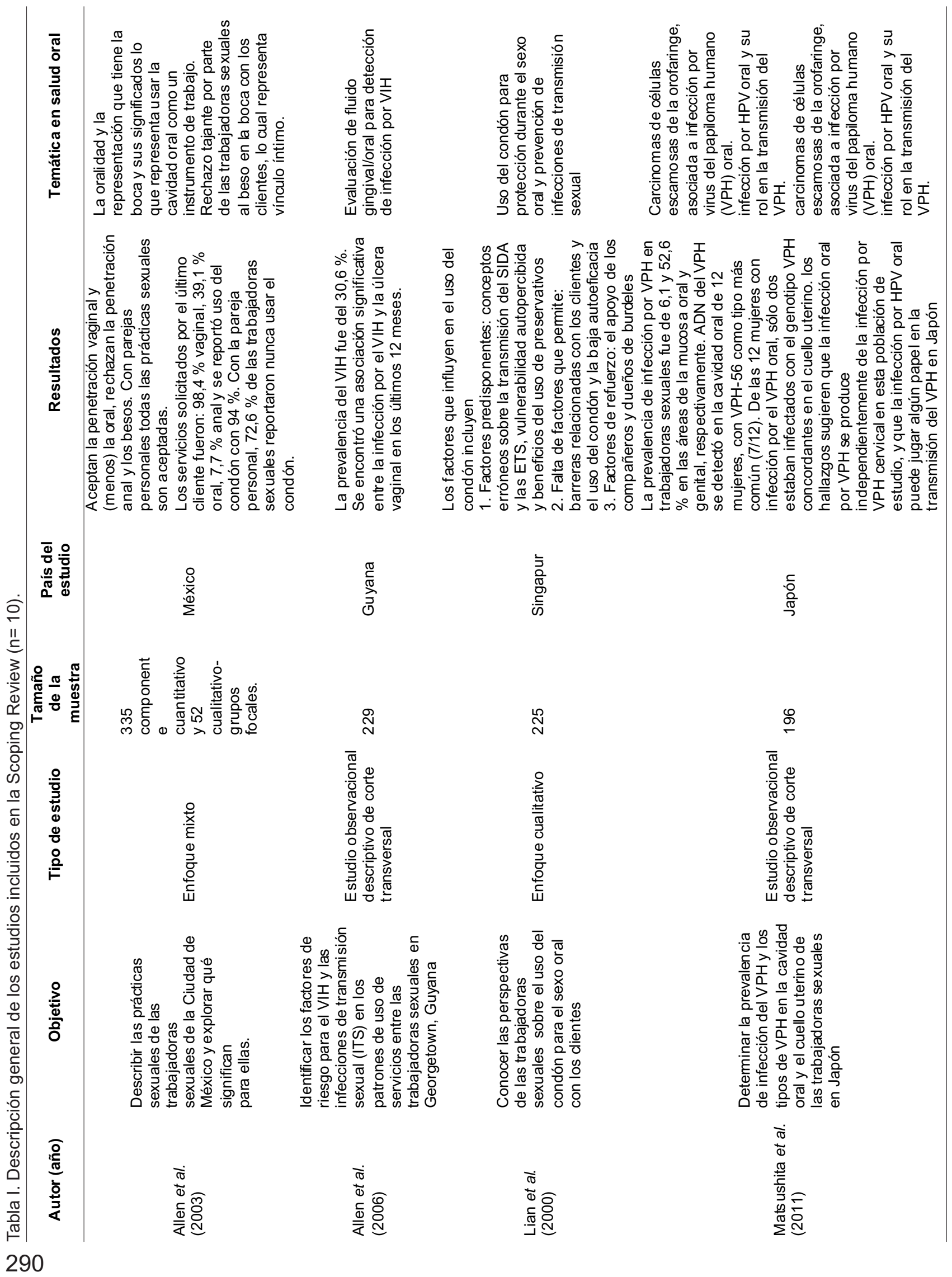



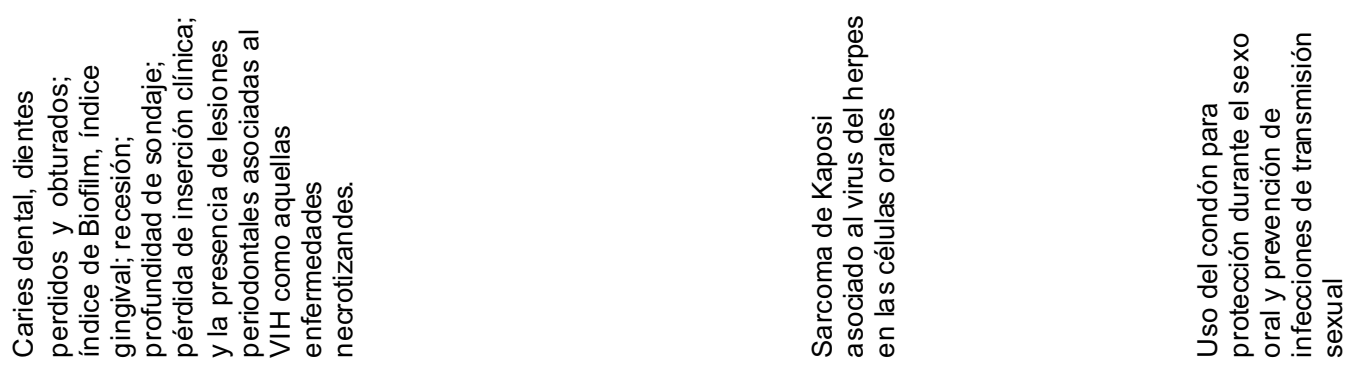

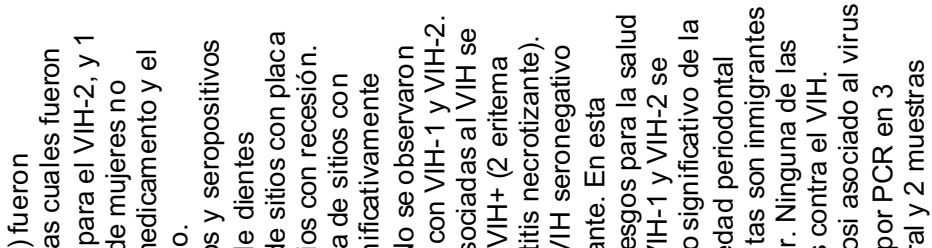

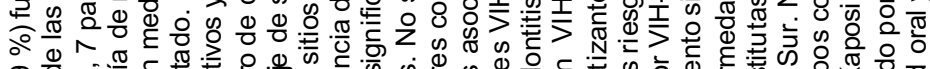

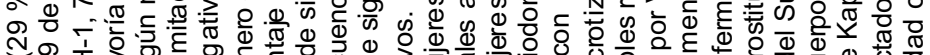
i

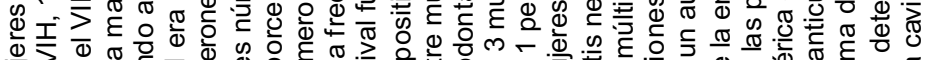

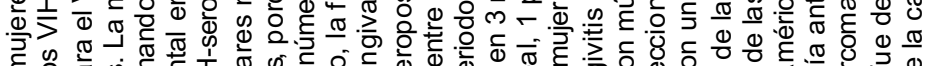

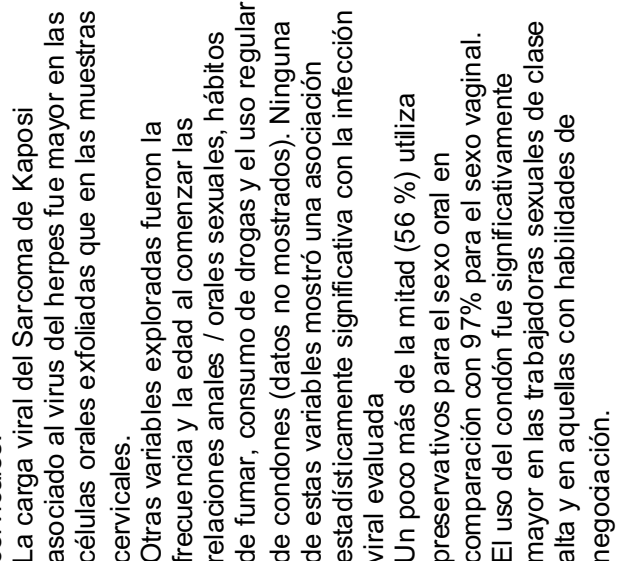

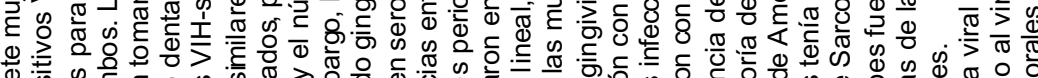

屯

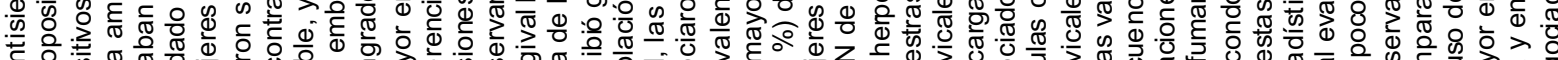

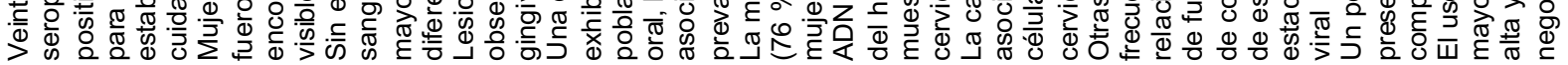

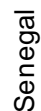

ช

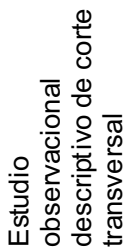

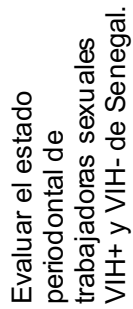

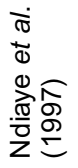

돈
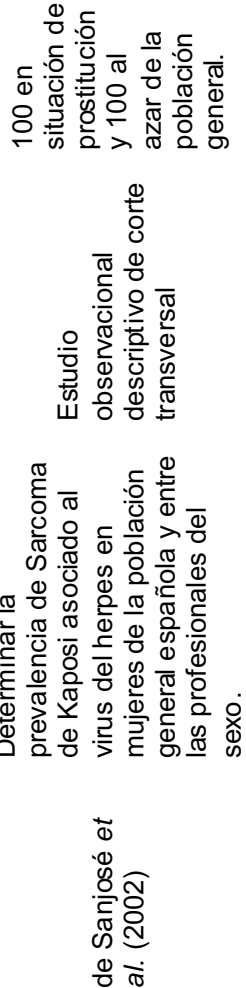

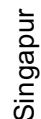
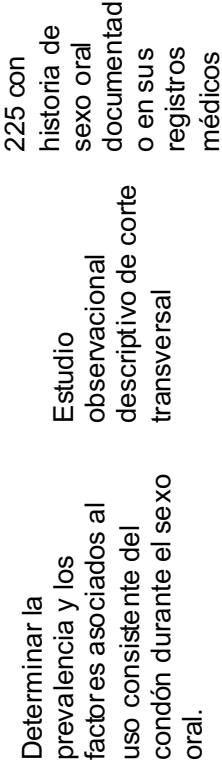


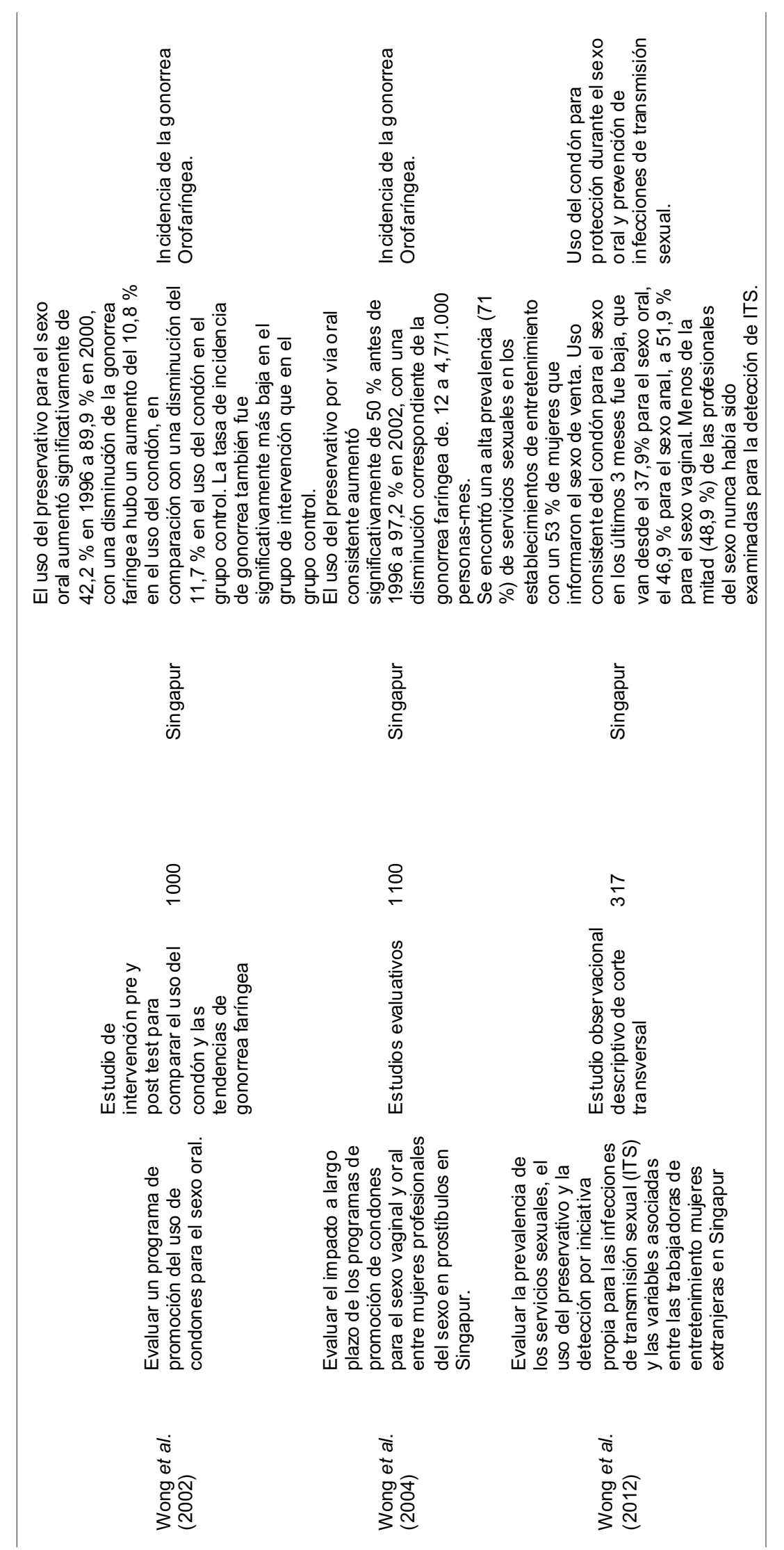

\section{DISCUSIÓN}

Los hallazgos principales de la Scoping Review dan cuenta de que a la fecha son escasos los estudios a nivel mundial en mujeres en situación de prostitución relacionados con salud oral. Adicionalmente se percibe algunas brechas de conocimiento relacionadas con la evaluacion de los determinantes relacionados con el proceso salud enfermedad oral, desde una perspectiva de la epidemiología social. No obstante, los resultados de los estudios incluidos desde diversos enfoques identifican múltiples factores de riesgo biológicos, asi como conductas y comportamiento para el desarrollo de patologías orales a los que se expone esta población. Hasta donde alcanza nuestro conocimiento, esta es una de las primeras revisiones sistemáticas exploratorias realizadas en esta población en el tema de interés.

Los resultados de este estudio brindan la oportunidad de visibilizar y reconocer que a la fecha existe un gran desconocimiento de aspectos biopsicosociales importantes de la vida, la salud, el cuidado y la identidad social de este colectivo especialmente vulnerable. En aspectos de acceso y oportunidad a los servicios de salud, los estudios revelan que las mujeres en situación de prostitución solo acceden a estos servicios para solicitar certificados que las acredite como mujeres aptas o sanas en términos de enfermedades de transmisión sexual, que les permita desempeñar su oficio y cuando requieren una atención prioritaria por presentar dichas patológicas (Amaya et al.).

De otro lado, importante considerar que los problemas relacionados con aspectos psicosociales, mentales y otras enfermedades dis- 
tintas a las infecciones de transmisión sexual y en especial del VIH/SIDA, son desconocidos y desatendidos lo cual contribuye con los aspectos de estigmatización social y discriminación a los que son sometidos este grupo (Sacks, 1996). Un estudio en mujeres en situación de prostitución en Colombia reveló en términos de discriminación y estigmatización social que el $6 \%$ de las mujeres encuestadas han percibido discriminación por parte de diferentes profesionales del área de salud; adicionalmente manifiestan ser discriminadas por sus vecinos, familiares, clientes, dueños de negocios, transeúntes personal de salud, fuerzas armadas. Un $50 \%$ de las trabajadoras sexuales mienten sobre su oficio y les da vergüenza incluso frente a personal de salud como médicos (Ministerio de la Protección Social). Estos aspectos que inciden en la calidad de vida de la mujer en esta situación de prostitución se explica en parte por fenómenos culturales que llevan a la mujer a desenvolverse en un mundo sin equidad de género, donde aún predomina la ideología patriarcal, y posición religiosa en posición a la autonomía de su sexualidad (Amaya et al.)

De acuerdo a los resultados encontrados se hace necesario el acercamiento al análisis de los procesos de salud enfermedad y su relación con la salud oral en esta población desde un enfoque que trascienda los componentes biológicos y fisiológicos, pues existen varios elementos implicados de naturaleza psicológica, económica, social y ambiental (Fernández et al., 2012). La propuesta de análisis de los determinantes sociales de la salud, ha hecho parte de las agendas y discusiones de la Organización Mundial de salud (OMS), como parte del cambio de paradigma curativo al enfoque de promoción de Salud y prevención de las enfermedades enmarcado en el logro de garantizar la salud como derecho fundamental en todos los países del mundo en especial en estas poblaciones con condiciones más desfavorable (Comisión de Determinantes Sociales, 2008; Borrell \& Malmusi, 2010).

Nuevos estudios desde la epidemiologia socíal deberían considerar aspectos relacionados con el estado de salud oral en las mujeres en situación de prostitución. Por ejemplo, se debe incluir la clase social evaluada en indicadores de ingresos, riqueza material y el nivel educativo (Muntaner et al., 2012). De igual manera, el acceso a los servicios de salud oral, las condiciones laborales, y la existencia de políticas sociales y económicas en un país son elementos cruciales para analizar la situación de vulnerabilidad social de diversos colectivos y que explican las desigualdades que en términos de salud se evidencian en las poblaciones
(Buss \& Pellegrini Filho, 2006). De igual manera, reconocer las particularidades de los grupos sociales implica condiderar las diferentes habilidades para la vida, los hábitos, los conocimientos y prácticas, los estilos de vida de cada persona además de las diferencias de sexo. Los estudios cualitativos cobran importancia para comprender las representaciones sociales frente a la salud oral (Stewart et al., 2008).

Se refleja la necesidad de formación de equipos de salud que asuman en su práctica respeto a los derechos sexuales y reproductivos en esta población, que además les permita tener una mirada sensible de lo que les sucede, a su vez facilitará replantear la oferta de los servicios de salud con base en las necesidades y la situación a la que se enfrentan día a día, con una perspectiva de género y centrada en el cuidado humano, fomentar redes de apoyo, de promoción de la salud y prevención de las enfermedades más prevalentes, sumado a la información sobre derechos, legislación para prevenir y atender la vulneración de sus derechos y su dignidad humana y la discriminación que ellas autoperciben por parte del personal de salud (Carter et al.; Amaya et al.; Ministerio de la Protección Social).

VÁSQUEZ, H. A.; VIVARES-BUILES, A. M. \&. AGUDELOSUÁREZ, A. A. The invisibility of oral health studies about women in situation of prostitution: A scoping review. Int. J. Odontostomat., 10(2):287-295, 2016.

ABSTRACT: This study aimed to describe the existing studies worldwide related to oral health in women in prostitution. For this exploratory review (Scoping Review) on the subject in question in studies published in the form of original scientific articles in journals peer review was conducted without restriction in the search period and indexed in 4 international databases (PubMed, EMBASE, LILACS and SCOPUS). Description of the main characteristics of the studies. The main results, after making the selection process 10 studies with different methodological approaches (quantitative, qualitative or mixed and intervention studies) were found. Six of the studies were conducted in Asian countries (Japan and Singapore). The themes were: oral manifestations of HIV, condom use, practices and attitudes towards oral sex. Only a specific oral health study conducted in Senegal which analyzes the periodontal situation of sex workers with and without HIV was found. In conclusion, studies on oral health for individuals in prostitution are scarce. Being a socially sensitive and vulnerable group, it is essential to encourage research in these women to identify potential situations of social inequality that impact oral health conditions in this group.

KEY WORDS: oral health, health profile, prostitution, dentistry research. 


\section{REFERENCIAS BIBLIOGRÁFICAS}

Agudelo Suárez, A. A. \& Martínez Herrera, E. La salud bucal colectiva y el contexto colombiano: un análisis crítico. Rev. Gerenc. Polit. Salud, 8(16):91-105, 2009.

Allen, B.; Cruz-Valdez, A.; Rivera-Rivera, L.; Castro, R.; Arana-García, M. E. \& Hernández-Avila, M. Afecto, besos y condones: el $\mathrm{ABC}$ de las prácticas sexuales de las trabajadoras sexuales de la Ciudad de México. Salud Pública Mex., 45(Supl. 5):S595-S607, 2003.

Allen, C. F.; Edwards, M.; Williamson, L. M.; Kitson-Piggott, W.; Wagner, H. U.; Camara, B. \& Hospedales, C. J. Sexually transmitted infection service use and risk factors for HIV infection among female sex workers in Georgetown, Guyana. J. Acquir. Immune Defic. Syndr., 43(1):96-101, 2006.

Amaya, A.; Canaval, G. E. \& Viáfara, E. Estigmatización de las trabajadoras sexuales: influencias en la salud. Colomb. Med., 36(3 Supl. 2):65-74, 2005.

Arksey, H. \& O'Malley, L. Scoping studies: towards a methodological framework. Int. J. Soc. Res. Methodol., 8(1):19-32, 2005.

Armstrong, R.; Hall, B. J.; Doyle, J. \& Waters, E. Cochrane Update. 'Scoping the scope' of a cochrane review. J. Public Health (Oxf.), 33(1):147-50, 2011.

Belza, M. J.; Clavo, P.; Ballesteros, J.; Menéndez, B.; Castilla, J.; Sanz, S.; Jerez, N.; Rodríguez, C.; Sánchez, F. \& del Romero, J. Condiciones sociolaborales, conductas de riesgo y prevalencia de infecciones de transmisión sexual en mujeres inmigrantes que ejercen la prostitución en Madrid. Gac. Sanit., 18(3):177-83, 2004.

Borrell, C. \& Malmusi, D. La investigación sobre los determinantes sociales y las desigualdades en salud: evidencias para la salud en todas las políticas. Informe SESPAS 2010. Gac. Sanit., 24(Supl. 1):101-8, 2010.

Buss, P. M. \& Pellegrini Filho, A. Iniqüidades em saúde no Brasil, nossa mais grave doença: comentários sobre o documento de referência e os trabalhos da Comissão Nacional sobre DeterminantesSociais da Saúde. Cad. Saúde Pública, 22(9):2005-8, 2006.

Carter, K. H.; Harry, B. P.; Jeune, M. \& Nicholson, D. Percepción del riesgo, comportamientos riesgosos y seroprevalencia del $\mathrm{VIH}$ en trabajadoras sexuales de Georgetown, Guyana. Rev. Panam. Salud Publica, 2(4):238-46, 1997.

Corte Constitucional. Sentencia T-629/10. Bogotá, Corte Constitucional, República de Colombia, 2010. Disponi- ble en: http://www.corteconstitucional.gov.co/relatoria/ 2010/t-629-10.htm

Ministerio de la Protección Social. Comportamiento Sexual y Prevalencia de VIH En Mujeres Trabajadoras Sexuales en Cuatro Ciudades de Colombia. Bogotá, Ministerio de la Protección Social, República de Colombia, 2008.

Comisión sobre Determinantes Sociales. Subsanar las desigualdades en una generación: Alcanzar la equidad sanitaria actuando sobre los determinantes sociales de la salud. Ginebra, Organización Mundial de la Salud, 2008. Disponible en: http://www.who.int/ social_determinants/final_report/ csdh_finalreport_2008_execsumm_es.pdf

de Sanjosé, S.; Marshall, V.; Solà, J.; Palacio, V.; Almirall, R.; Goedert, J. J.; Bosch, F. X. \& Whitby, D. Prevalence of Kaposi's sarcoma-associated herpesvirus infection in sex workers and women from the general population in Spain. Int. J. Cancer, 98(1):155-8, 2002.

Fernández, R. M.; Thielmann, K. \& Bormey Quiñones, M. B. Determinantes individuales y sociales de salud en la medicina familiar. Rev. Cuba. Salud Pública, 38(3):484-90, 2012.

International Committee of Medical Journal Editors (ICMJE). Recommendations for the Conduct, Reporting, Editing, and Publication of Scholarly Work in Medical Journals. International Committee of Medical Journal Editors (ICMJE), 2015. Disponible en: http://www.icmje.org/.

Juliano, D. El trabajo sexual en la mira: polémicas y estereotipos. Cad. Pagu, (25):79-106., 2005

Lian, W. M.; Chan, R. \& Wee, S. Sex workers' perspectives on condom use for oral sex with clients: a qualitative study. Health Educ. Behav., 27(4):502-16, 2000.

Manchado Garabito, R.; Tamames Gómez, S.; López González, M.; Mohedano Macías, L. \& Veiga de Cabo, J. Revisiones sistemáticas exploratorias. Med. Segur. Trab., 55(216):12-9, 2009.

Matsushita, K.; Sasagawa, T.; Miyashita, M.; Ishizaki, A.; Morishita, A.; Hosaka, N.; Saikawa, K.; Hoshina, S.; Bi, X. \& Ichimura, H. Oral and cervical human papillomavirus infection among female sex workers in Japan. Jpn. J. Infect. Dis., 64(1):34-9, 2011.

Muntaner, C.; Rocha, K. B.; Borrell, C.; Vallebuona, C.; Ibáñez, C.; Benach, J. \& Sollar, O. Clase social y salud en América Latina. Rev. Panam. Salud Pública, 31(2):166-75, 2012. 
Ndiaye, C. F.; Critchlow, C. W.; Leggott, P. J.; Kiviat, N. B.; Ndoye, I.; Robertson, P. B. \& Georgas, K. N. Periodontal status of HIV-1 and HIV-2 seropositive and HIV seronegative female commercial sex workers in Senegal. J. Periodontol., 68(9):827-31, 1997.

Newton, J. T. \& Bower, E. J. The social determinants of oral health: new approaches to conceptualizing and researching complex causal networks. Community Dent. Oral Epidemiol., 33(1):25-34, 2005.

Petersen, P. E. \& Kwan, S. Equity, social determinants and public health programmes--the case of oral health. Community Dent. Oral Epidemiol., 39(6):481-7, 2011.

Ribando, S. C. Trafficking in Persons in Latin America and the Caribbean. Washington D. C., Congressional Research Service, 2010. Disponible en: http:// digitalcommons.ilr.cornell.edu/key_workplace/792

Rúa Serna, J. C. El derecho a prostituirse. La prostitución a la luz del derecho laboral. Diálogos Derecho Polit., 9(3):111, 2012.

Sacks, V. Women and AIDS: an analysis of media misrepresentations. Soc. Sci. Med., 42(1):59-73, 1996.

Stewart, K.; Gill, P.; Chadwick, B. \& Treasure, E. Qualitative research in dentistry. Br. Dent. J., 204(5):235-9, 2008.

Wong, M. L.; Chan, R. \& Koh, D. Long-term effects of condom promotion programmes for vaginal and oral sex on sexually transmitted infections among sex workers in Singapore. AIDS, 18(8):1195-9, 2004.

Wong, M. L.; Chan, R.; Tan, H. H.; Yong, E.; Lee, L.; Cutter, J.; Tay, J. \& Koh, D. Sex work and risky sexual behaviors among foreign entertainment workers in urban Singapore: findings from Mystery Client Survey. J. Urban Health, 89(6):1031-44, 2012.

Wong, M. L.; Chan, R. K. \& Koh, D. Promoting condoms for oral sex: impact on pharyngeal gonorrhea among female brothel-based sex workers. Sex. Transm. Dis., 29(6):3118, 2002.

Wong, M. L.; Chan, R. K.; Koh, D. \& Wee, S. Factors associated with condom use for oral sex among female brothel-based sex workers in Singapore. Sex. Transm. Dis., 27(1):39-45, 2000.
Dirección para Correspondencia:

Alejandro Vásquez Hernández

Facultad de Odontología

Fundación Universitaria Autónoma de las Américas

Calle 34A No $76-35$

Medellín

COLOMBIA

Email: alevhz@gmail.com

Recibido: 23-07-2015

Aceptado: 14-04-2016 